\title{
The Operation of Detection Systems in Conditions of Contrast Decrease of Ground Objects
}

\author{
Stanislav Trehubenko ${ }^{1}$, Lubov Berkman ${ }^{2}$, NataliiaYeromina $^{3}$, Sergey Petrov $^{4}$, Yevhen Bryzhatyi $^{5}$, \\ Hennadii Kovalov ${ }^{6}$, Volodymyr Dachkovskyi ${ }^{7}$, Liudmyla Mikhailova ${ }^{8}$ \\ ${ }^{1}$ Central Research Institute of the Armed Forces of Ukraine, Ukraine, Stas.trigub@icloud.com \\ ${ }^{2}$ Department of Telecommunication Systems and Networks, State University of Telecommunications, Ukraine, \\ 090289@ukr.net \\ ${ }^{3}$ Department of Electronic Computers, Kharkiv National University of Radio Electronics, Ukraine, Kharkiv, \\ nataliia.yeromina@nure.ua \\ ${ }^{4}$ Department of Physics, Electrical Engineering and Power Engineering, Ukrainian Engineering Pedagogics \\ Academy, Ukraine, psv_topol@ukr.net \\ ${ }^{5}$ Department of Military training, Kamianets-Podilskyi National Ivan Ohiienko University, Ukraine, evgen- \\ 5335@ukr.net \\ ${ }^{6}$ Department of tactics, combat (operational) units, Hetman Petro Sahaidachny National Army Academy, \\ Ukraine, inginer1@ukr.net \\ ${ }^{7}$ National Ivan Cherniakhovsky Defense University of Ukraine, Ukraine, 1903vova@ukr.net \\ ${ }^{8}$ Department of Energy and Electrical Systems of the Agro-industrial Complex, State Agrarian and Engineering \\ University in Podilia, Ukraine, mihajlovaimesg@ gmail.com
}

\begin{abstract}
The presented results refer the generalized assessment of the effectiveness of the ground objects detection systems' operation based on the tracking and comparison method. The generalized relation for the definition of the ground objects detection probability was received, without the link to the specific detection system sensor's type and in accordance with the objective function, formed by the system. The relations, which were deduced for the asymptotic estimate of the probability of position measurement of the detection system, allow us to identify the confidence interval of the required probability. The results of the research on the objective function with the help of the informative parameters of the detection system are presented. The main operation criteria in conditions of decreasing ground objects' contrast are identified when using the radiometric detection system. The simulation results of the ground objects detection by the radiometric systems in conditions of decreasing radio-thermal contrasts are presented.
\end{abstract}

Key words: detection systems, ground objects, objective function, detection probability, radio-thermal contrasts.

\section{INTRODUCTION}

The information on the ground objects, which is received with the help of the detection systems, based on the tracking and comparison method, is mainly defined by the primary conversion sensor. Depending on the tasks of the detection system, the separate optical-electronic, radar, radiometric sensors as well as their combinations can be utilized [1-4]. However, regardless of the type of sensors, which are collecting the data on the ground objects, the effectiveness of the detection systems' operation is mainly defined by the parameters of the detected objects, which consequently specify the probability of their search and detection.
Moreover, in number of cases the certain measures can be applied to reduce the level of radar and radiometric visibility with the help of the specific coatings and materials.

\subsection{Problem analysis}

The results of the theoretical research, performed by the authors in range of search, detection and identification of the ground objects against various backgrounds in order to define the proper positioning data of the detection system, indicate the necessity of the research targeted on the evaluation and improvement of the effectiveness of the detection systems' operation in conditions of adoption of measures on the decline in their efficiency [1-5]. Taking into consideration the variation of sensors for acquisition of information regarding the ground objects, the generalized evaluation of the effectiveness of the detection systems must be performed regardless of the type of sensor, the conditions of the disruption of operation must be identified, the ways of improvement of the effectiveness need to be defined.

The aim of the article is the generalized evaluation of the effectiveness of the ground objects detection systems' operation, based on the tracking and comparison method of the information extraction, and the definition of the ways of improvement of their effectiveness in conditions of decrease of the information contrasts.

\section{MAIN MATERIAL}

2.1The definition of the asymptotic estimate of the probability of position measurement of the ground objects detection system in stochastic conditions

The solving of the problem of detection of particular objects or their groups in defined coordinates with the help of detection systems in question is based on the comparison of 
the received information with the prior one. Based on the afore-mentioned and taking into account the stochastic nature of information on the detected object, it is advisable to use the detection probability (DP) $-\mathrm{P}_{\mathrm{D}}$, which will be defined in accordance with the theory of estimation.

According to the chosen approach to solving of the problem of detection of the ground object against the corresponding background, the subjects are: the differentiation of statistic hypothesis $\mathrm{H}_{0}$, the lack of informative parameter $\Delta \mathrm{T}$ contrast in the analyzed resolution cell from alternative $\mathrm{H}_{1}$, which corresponds to the contrast availability. The informative parameter evaluation is the statistical estimate of noise distribution parameter, recorded at the output of the corresponding informative parameter sensor.

The complexity of the reviewed tasks lays in the prior uncertainty due to unspecified background parameters as well as to the instability of the detection system sensors' characteristics. Using the specified parameters of the reviewed selection of reading $u_{i}$ of the noise $U(t)$ at the sensor's output, the likelihood ratio $\Lambda$ can be represented as the relation between the probability of belonging of the data to the alternative $\mathrm{H}_{1}$ and the hypothesis $\mathrm{H}_{0}$, as well as the $\operatorname{logarithm} \mathrm{Z}$, used for the optimum statistic for this relation, can be calculated. Hence, using the specified selection range, the comparison with the threshold $\mathrm{C}$ in case of $\mathrm{Z} \geq \mathrm{C}$ displays the alternative $\mathrm{H}_{1}$ as preferable, while the comparison with the threshold $\mathrm{C}$ in case of $\mathrm{Z}<\mathrm{C}$ displays the hypothesis $\mathrm{H}_{0}$ as more preferable. In consideration of the foregoing, the task of the ground object detection will be solved based on the assumption that the detection sensor forms the certain matrix of the informative parameter measurements $A_{1} \times A_{2}$. Moreover, the preformed reference informative parameter is expressed as matrix with the dimensions $B_{1} \times B_{2}$, where $B_{1}<A_{1}, B_{2}<A_{2}$. The sensor's internal noise value $n$ is considered as additive noise with the zero average and dispersion $\sigma_{i j}^{2}$. In should be taken into consideration that in case of limited time frame for signals $\tau$ collection by the detection system, the noise distribution law should be applied as regular.

According to the aforementioned, let us analyze the objective function as a result of the comparison of matrix $\mathrm{A}_{1} \times \mathrm{A}_{2}$ and $\mathrm{B}_{1} \times \mathrm{B}_{2}$. For simplicity, let us analyze the measurements of the objective function $R\left(1_{i}\right)$ as onedimensional in $\mathrm{N}$ points $\mathrm{l}_{\mathrm{i}}=\mathrm{i} \tau_{\mathrm{k}}\left(\tau_{\mathrm{k}}-\right.$ correlation window $)$. As a result the measurement of the objective function $\mathrm{R}\left(\mathrm{l}_{\mathrm{i}}\right)=\mathrm{R}\left(\mathrm{i} \tau_{\mathrm{k}}\right)$ in the described points will be statistically independent, while one of the $\mathrm{N}$ points will coincide with the theoretical variable of the parameter $1_{0}$. Based on the aforementioned, the DP of the ground object with the known coordinates can be represented as follows:

$$
\mathrm{P}_{\mathrm{D}}=\mathrm{P}[\mathrm{R}(\mathrm{k})>\mathrm{R}(\mathrm{i}), \mathrm{i}=1,2, \ldots \mathrm{N}, \mathrm{i} \neq k],
$$

where $\mathrm{N}=\frac{\mathrm{L}}{\Delta_{\mathrm{L}}}+1$ is the quantity of lateral emissions of the objective function;

$\mathrm{L}$ is the prior interval of values of the objective function.
Let us represent the function of the detection system's objective function distribution in the absolute point as $f_{R(k)}(r)$, while the similar function for the lateral emissions as $f_{R(i)}(\dot{r})$. As a result the relation for the definition of the ground object DP is represented as follows:

$$
\mathrm{P}_{\mathrm{D}}=\int_{-\infty}^{\infty} \prod_{\substack{\mathrm{i}=1 \\ \mathrm{i} \neq \mathrm{k}}}^{\mathrm{N}}\left[\int_{-\infty}^{\mathrm{R}} \mathrm{f}_{\mathrm{R}(\mathrm{i})}(\mathrm{y}) \mathrm{dy}\right] \mathrm{f}_{\mathrm{R}(\mathrm{k})}(\mathrm{R}) \mathrm{dR}
$$

In expression (2) the following notation is used:

$$
\prod_{\substack{i=1 \\ i \neq k}}^{N} \int_{-\infty}^{R} f_{R(i)}(y) d y=P_{N}(R) .
$$

Taking into account the assumption that the values $\mathrm{R}(\mathrm{i})$ and $\mathrm{R}(\mathrm{k})$ are independent and follow the regular probability distribution law, not considering the intermediate conversions, the final expression for the DP and, consequently, position measurement of the detection system will be represented as follows:

$$
\begin{aligned}
\mathrm{P}_{\mathrm{D}}= & \frac{1}{\sqrt{2 \pi \sigma_{\mathrm{c}}^{2}}} \int_{-\infty}^{\infty} \exp \left\{-\frac{[\mathrm{y}-<\mathrm{R}(\mathrm{k})>]^{2}}{2 \sigma_{\mathrm{c}}^{2}}\right\} \times \\
& \times \prod_{\substack{\mathrm{i} \neq \mathrm{k} \\
\mathrm{i}=1}}^{\mathrm{N}} \Phi\left[\frac{\mathrm{y}-<\mathrm{R}(\mathrm{i})>}{\sigma_{\mathrm{n}}}\right] \mathrm{dy},
\end{aligned}
$$

where $\sigma_{\mathrm{c}}^{2}$ is the dispersion of values of the objective function $\mathrm{R}(\mathrm{k})$;

$\langle\mathrm{R}(\mathrm{i})\rangle=\mathrm{M}[\mathrm{R}(\mathrm{i})] ;\langle\mathrm{R}(\mathrm{k})\rangle=\mathrm{M}[\mathrm{R}(\mathrm{k})]$ are the expected values of objective function $\mathrm{R}$ in the corresponding points; $\sigma_{\mathrm{n}}^{2}$ is the dispersion of values of the objective function $\mathrm{R}(\mathrm{i})$; $\Phi(\mathrm{x})$ is the probability integral.

The relation (4) doesn't take into the consideration the stochastic nature of the included parameters. Consequently it's imperative to define the confidence interval of the probability of position measurement of the detection system $\left\{\mathrm{P}_{\mathrm{D} \text { min }}, \mathrm{P}_{\mathrm{Dmax}}\right\}$. The values $\mathrm{P}_{\mathrm{D} \text { min }}$ and $\mathrm{P}_{\mathrm{D} \max }$ can be calculated after the evaluation of the solution tendencies while $\sigma_{\mathrm{c}} \rightarrow 0$ and $\sigma_{\mathrm{n}} \rightarrow \infty$, recording $\sigma_{\mathrm{n}}$ and $\sigma_{\mathrm{c}}$ respectively. This can be achieved using the Laplace's method for the integral asymptotic estimate [6].

The $\sigma_{\mathrm{c}}$ needs to be fixed and after that the behavior of the integral included into (4) at $\sigma_{\mathrm{c}} \rightarrow 0$ can be examined. Thus the following notions are used: 
Stanislav Trehubenko et al., International Journal of Emerging Trends in Engineering Research, 8(1), January 2020,208 - 212

$$
\begin{gathered}
\mathrm{F}(\mathrm{y})=\prod_{\substack{\mathrm{i} \neq \mathrm{k} \\
\mathrm{i}=1}}^{\mathrm{N}} \Phi\left[\frac{\mathrm{y}-<\mathrm{R}(\mathrm{i})>}{\sigma_{\mathrm{n}}}\right], \\
\lambda=\frac{1}{2 \sigma_{\mathrm{c}}^{2}}
\end{gathered}
$$

According to (5) and (6) it is required to investigate the behavior of the integral (4) and receive its asymptotic estimate at $\lambda \rightarrow \infty$. Taking into account the smooth changes patters of the functions $\mathrm{F}(\mathrm{y})$ (in comparison to the exponential factor) and $\delta$-typed pf the first factor, the integral (4) rearranges to the following form:

$$
\begin{aligned}
& \mathrm{P}_{\mathrm{D}}=\int_{-\infty}^{+\infty} \mathrm{e}^{-\lambda(\mathrm{y}-<\mathrm{R}(\mathrm{k}))^{2}} \mathrm{~F}(\mathrm{y}) \mathrm{dy} \approx \\
& \approx \mathrm{F}(\mathrm{R}(\mathrm{k})) \int_{-\infty}^{+\infty} \mathrm{e}^{-\lambda(\mathrm{y}-<\mathrm{R}(\mathrm{k}))^{2}} \mathrm{dy} .
\end{aligned}
$$

In (7) let us make the transposition as follows:

$$
\sqrt{\lambda \times}(\mathrm{y}-\langle\mathrm{R}(\mathrm{k})\rangle)=\mathrm{t}, \quad \mathrm{dy}=\frac{\mathrm{dt}}{\mathrm{d} \sqrt{\lambda}} .
$$

Taking into account the performed transposition, the expression (6) is:

$$
\begin{aligned}
& \mathrm{P}_{\mathrm{D}} \approx \mathrm{F}(\mathrm{R}(\mathrm{k})) \cdot \frac{1}{\sqrt{\lambda}} \int_{-\infty}^{+\infty} \mathrm{e}^{-\mathrm{t}^{2}} \mathrm{dt}= \\
& =\mathrm{F}(\mathrm{R}(\mathrm{k})) \cdot \sqrt{2} \cdot \sigma_{\mathrm{c}} \frac{\sqrt{\pi}}{2}
\end{aligned}
$$

As a result, the asymptotic estimate of the probability of position measurement of the ground objects detection system can be represented as:

$$
\begin{aligned}
& \mathrm{P}_{\mathrm{D}_{\sigma_{\mathrm{c}} \rightarrow 0}} \approx \sqrt{\frac{\pi}{2}} \sigma_{\mathrm{c}} \mathrm{F}(\mathrm{R}(\mathrm{k}))+0\left(\sigma_{\mathrm{c}}\right) \approx \\
& \approx \sqrt{\frac{\pi}{2}} \sigma_{\mathrm{c}} \prod_{\mathrm{i}=1}^{\mathrm{N}} \Phi\left(\frac{<\mathrm{R}(\mathrm{k})>-<\mathrm{R}(\mathrm{i})>}{\sigma_{\mathrm{n}}}\right)+0\left(\sigma_{\mathrm{c}}\right) . \\
& \mathrm{i} \neq \mathrm{k}
\end{aligned}
$$

Using the law $3 \sigma$ [7], the expression (6) is transformed into:

$$
\begin{aligned}
& \mathrm{P}_{\mathrm{D}} \approx \frac{1}{\sqrt{2 \pi} \sigma_{\mathrm{c}}} \mathrm{R}(\mathrm{k})+\mathrm{A}(\mathrm{k})-\mathrm{A} \sqrt{2} \sigma_{\mathrm{c}} \sigma_{\mathrm{c}} \mathrm{e}^{-\frac{(\mathrm{y}-<\mathrm{R}(\mathrm{k})>)^{2}}{2 \sigma_{\mathrm{c}}^{2}}} \times \\
& \times \prod_{\mathrm{i} \neq \mathrm{k}}^{\mathrm{N}} \Phi\left(\frac{\mathrm{y}-<\mathrm{R}(\mathrm{i})>}{\sigma_{\mathrm{n}}}\right) \mathrm{dy} . \\
& \mathrm{i}=1
\end{aligned}
$$

In expression (10) $\mathrm{A}=3 \ldots 4$.

We should keep in mind that the value varies within the given range and $\sigma_{\mathrm{n}} \rightarrow \infty$. Hence, the factor for the probability integral, included into (10) can be roughly represented as follows:

$$
\Phi\left(\frac{\mathrm{y}-\langle\mathrm{R}(\mathrm{i})\rangle}{\sigma_{\mathrm{n}}}\right)_{\sigma_{\mathrm{n}} \rightarrow \infty} \approx \frac{1}{2}+\frac{1}{\sqrt{2 \pi}} \frac{\mathrm{y}-\langle\mathrm{R}(\mathrm{i})\rangle}{\sigma_{\mathrm{n}}}
$$

Taking into account (11) the finite expression for the asymptotic estimate of the probability of position measurement of the ground objects detection system will be determined as follows:

$$
\begin{aligned}
& \mathrm{P}_{\mathrm{D}_{\mathrm{n}} \rightarrow \infty} \approx{\frac{1}{\sqrt{2 \pi} \sigma_{\mathrm{c}}}}_{\mathrm{R}(\mathrm{k})-\mathrm{A} \sqrt{2} \sigma_{\mathrm{c}}}^{\mathrm{R}(\mathrm{k})+\mathrm{A} \sqrt{2} \sigma_{\mathrm{c}}} \int^{-\frac{(\mathrm{y}-<\mathrm{R}(\mathrm{k})>)^{2}}{2 \sigma_{\mathrm{c}}^{2}}} \times
\end{aligned}
$$

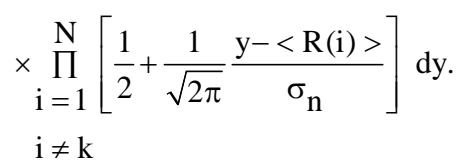

The relation (12) allows evaluating the asymptotic estimate of the probability of position measurement of the ground objects detection system at $\sigma_{\mathrm{n}} \rightarrow \infty$ using the known numerical characteristics of the objective function.

2.2 The definition of numerical characteristics of the objective function with the help of informative parameters of the detection system

The definition of numerical characteristics of the objective function with the help of informative parameters of the detection system will be carried out as the linear approximation. Use of the mentioned assumption gives us the opportunity to present the objective function of the detection system with the help of its pulse-response characteristic $\mathrm{S}(\mathrm{t}+\tau)$.

In the first approximation, the objective function can be represented using the object reference informative parameter $\mathrm{x}(\mathrm{t})$ as follows: 
Stanislav Trehubenko et al., International Journal of Emerging Trends in Engineering Research, 8(1), January 2020,208 - 212

$$
R(\tau)=\int_{L} x(t) S(t+\tau) d t
$$

where $\mathrm{L}$ is the integration domain, which is defined by the linear size either of the reference informative parameter or of the received informative parameter. The lesser values are selected.

The received output informative parameter will be represented as follows:

$$
\mathrm{S}(\mathrm{t})=\mu \varepsilon(\mathrm{t}) \times(\mathrm{t})+\xi(\mathrm{t})
$$

where $\mu \in 0 \ldots 1$ is the coefficient, which characterizes the level of margin between reference and received informative parameters;

$\varepsilon(\mathrm{t})=\varepsilon_{0}+\delta \varepsilon(\mathrm{t})=\varepsilon_{0}[1+\dot{\varepsilon}(\mathrm{t})]$-the multiplicative noises, which are defined by the environment for signal propagation of the detection system;

$\xi(\mathrm{t})=\mathrm{n}(\mathrm{t})+\mathrm{v}(\mathrm{t})$ - the additive noises of the detection system sensor with regards to the background component $v(t)$, which distorts the informative signal.

Therefore, the expression (13) can be presented for the objective function as follows:

$$
\begin{aligned}
& R(\tau)=\int_{L} \mu \varepsilon_{0}[1+\dot{\varepsilon}(t)] x(t) x(t+\tau) d t+ \\
& \quad+\int_{L} x(t)[n(t+\tau)+v(t+\tau)] d t .
\end{aligned}
$$

It is clear that the expression (15) for the objective function includes both the informative and noise components that allows to formulate the following generalized expression:

$$
\mathrm{R}(\tau)=\mathrm{R}_{\mathrm{c}}(\tau)+\mathrm{R}_{\mathrm{n}}(\tau)
$$

where $R_{c}(\tau)$ is the useful part of the function;

$R_{n}(\tau)$ - noise part of the function.

Thus, identifying the useful and noise parts of the objective function for the detection system, its general numerical characteristics can be described.

According to the formula (15) and (16):

$$
\begin{gathered}
\left\langle\mathrm{R}_{\mathrm{c}}(\tau)\right\rangle=\mathrm{L} \mu \varepsilon_{0} \mathrm{R}_{\mathrm{x}}(\tau)=\mathrm{L} \mu \varepsilon_{0} \rho_{\mathrm{x}}(\tau) \rho_{\varepsilon}(\tau), \\
\left\langle\mathrm{R}_{\mathrm{n}}(\tau)\right\rangle=0 \\
\sigma_{\mathrm{c}}^{2}=\left\langle\mathrm{R}_{\mathrm{c}}^{2}(\tau)\right\rangle-\left\langle\mathrm{R}_{\mathrm{c}}(\tau)\right\rangle^{2}
\end{gathered}
$$

$$
\sigma_{\mathrm{n}}^{2}=\left\langle\mathrm{R}_{\mathrm{n}}^{2}(\tau)\right\rangle
$$

The relations (19) and (20) describe the variability of values of the objective function in the domain of ideal values and lateral emissions (Formula 4).

Considering the foregoing presentation of the multiplicative and additive noises, the expressions (19) and (20) can be represented as follows:

$$
\begin{gathered}
\sigma_{\mathrm{c}}^{2}=\mathrm{P}_{\mathrm{x}} \mu^{2}{ }_{0}^{2} \rho_{\varepsilon}(\tau) \rho_{\mathrm{x}}(\tau) \mathrm{L}^{2}+ \\
+\mu_{0}^{2} \varepsilon_{0}^{2}\left[1+\rho_{\dot{\varepsilon}}(\tau)\right] \cdot 2 \frac{\Delta \mathrm{F}}{\Delta \mathrm{f}}\left[1+\rho_{\mathrm{x}}(\tau)\right] \mathrm{P} \\
\sigma_{\mathrm{n}}^{2}=2 \frac{\Delta \mathrm{F}}{\Delta \mathrm{f}}\left(\mathrm{P}_{\mathrm{X}}^{\mathrm{P}}+\mathrm{P}_{\mathrm{X}} \mathrm{P} v\right),
\end{gathered}
$$

where $P_{i}$ is the power of useful and noise components of the signal's informative parameters;

$\rho_{X}(\tau), \rho_{\varepsilon}(\tau)-$ are the cross-correlation coefficients of the useful and noise components of the signal's informative parameters;

$\Delta \mathrm{F}$ - transition band for the low-frequency part of the detection system sensor ;

$\Delta \mathrm{f}$ - transition band for the high-frequency part of the detection system sensor.

Defined general numerical characteristics of the objective function, received with the help of the informative parameters of the detection system signals, allow us to evaluate the asymptotic estimate of the probability of position measurement of the ground objects detection system.

2.3 The results of the numerical estimate of the probability of position measurement of the ground objects detection system

In accordance with the developed approach to the assessment of the effectiveness of the ground objects detection system the general numerical calculations for the DP of the ground object were performed, based on the signal-to-noise ratio $\mathrm{q}$ at the output of the detection system and on the quantity of the lateral emissions of the objective function $\mathrm{N}$. The results of the numerical estimate are shown in Figure 1. 


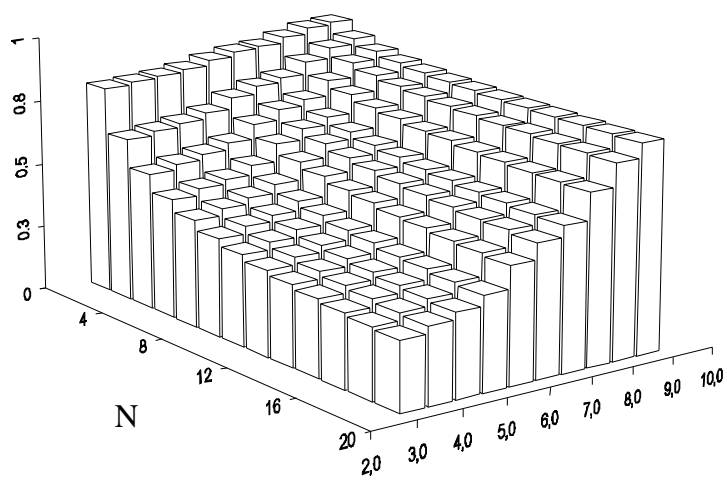

Figure 1: The probability of position measurement of the object detection systembased on the output signal-to-noise ratio and the quantity of the lateral emissions of the objective function.

The analysis of the diagram, displayed in the Fig. 1 displays that the increase of the useful component of the signal, which implies the greater quantity of the lateral emissions, results in the decreasing of the object DP.

As exemplified by the radiometric detection system, the reduction of the radiometric visibility of the ground objects corresponding to the decrease of the radio-thermal contrasts till $20-60 \mathrm{~K}$ results in the reduction of the signal-to-noise ratio to $\mathrm{q} 2 \ldots 4$ and in the increase of the lateral emissions of the objective function up to 10 . This results in the considerable decrease of the ground objects DP and the decrease of the probability of position measurement of the system itself to $0,15 \ldots 0,20$ respectively. Thus, because of the performed research, the conditions of the decrease of the effectiveness of the detection systems' operation in conditions of the reduction of radiometric visibility of the ground objects.

\section{CONCLUSION}

As a result of the performed research on the generalized evaluation of the effectiveness of the ground objects detection systems' operation, based on the tracking and comparison method of the information extraction, the generalized relation for the definition of the DP of ground objects, depending on the formed system of objective function, was receivedThe relations for the asymptotic estimate of the probability of position measurement of the detection system were received, which allow to identify the confidence interval of the required probability. As exemplified by the radiometric system, the reduction of the radiometric visibility of the ground objects corresponding to $20-60 \mathrm{~K}$ results in the massive reduction of the ground objects DP and the probability of position measurement of the detection system up to $0,15 \ldots 0,20$ respectively.

As a result of the research on the objective function using the detection system characteristics, it is displayed that the decrease of the information contrast characteristic such as radiometric visibility of the ground objects in particular, can result in the increase of the lateral emissions of the objective function up to 10 .
The derived estimates are the conditions of the decrease of the effectiveness of the detection systems' operation in conditions of the reduction of radar and radiometric visibility of the ground objects. These estimates can be used as conditions of countermeasures against such systems.

\section{REFERENCES}

1. V. Antyufeev. Matrix radiometric correlation-extreme navigation systems for aircraft: monographUkraine, Kharkov: KhNU V.N. Karazin, 372 p.

2. A. Sotnikov, V. Tarshyn, N. Yeromina, S. Petrov, N. Antonenko. A method for localizing a referenceobject in a current image with several bright objects, Eastern-European Journal of EnterpriseTechnologies. 2017, Vol. 3. № 9 (87), pp. 68-74. DOI: 10.15587/17294061.2017.101920.

3. O. Vorobiov, A. Sotnicov, ATantsiura. Models ofcurrent images that are formed by the combined correlation-extremal navigation system of a nonflammable aircraft. Modern information technologiesin the sphere of security and defence. Ukraine, Kyiv, - 2018, Vol.2, No.32. - pp. 29-36.

4. N.Yeromina, S. Petrov, A. Tantsiura, M. Iasechko, V. Larin. Formation of reference images and decisionfunction in radiometric correlation-extremal navigation systems. Eastern-European Journal ofEnterprise Technologies. - 2018, Vol.4, No.9 (94). pp. 27-35. DOI: $10.15587 / 1729-4061.2018 .139723$

5. Kulikov E. I., Trifonov A. P. Estimation of signal parameters against the background of interference.M.: Sov. radio, 1978. - 296 p.

6. Fedoryuk M. V. Asymptotics, Integrals and series, 1987. - 363p.

7. Gubar L. N., Ermolenko A.V. Probability theory and mathematical statistics: educational manual / L. N. Gubar, A.V. Ermolenko. - Syktyvkar: Publishing house SGU imeni pitirima Sorokina, 2015 - 120 p. 